\title{
Chemotherapy for T1 pNOM0 Breast Cancer Patients: A Propensity Score Matching Study Based on SEER Database and External Data
}

\section{Kaiwen Shen}

Traditional Chinese Medicine Hospital of Kunshan

Longdi Yao

Traditional Chinese Medicine Hospital of Changxing

\section{Huihua Cao}

Traditional Chinese Medicine Hospital of Kunshan

\section{Ximing Gu}

Traditional Chinese Medicine Hospital of Kunshan

Jie Wang

Traditional Chinese Medicine Hospital of Kunshan

\section{Jingyuan Zhu}

Traditional Chinese Medicine Hospital of Kunshan

\section{Deyuan Fu ( 061106333@fudan.edu.cn )}

Northern Jiangsu People's Hospital https://orcid.org/0000-0003-3636-399X

\section{Primary research}

\section{Keywords:}

Posted Date: March 19th, 2021

DOI: https://doi.org/10.21203/rs.3.rs-304571/v1

License: @ (i) This work is licensed under a Creative Commons Attribution 4.0 International License. Read Full License 


\section{Abstract \\ Background}

There is no definitive, unified view on chemotherapy for T1 pNOM0 breast cancer. Our study explored the effects of chemotherapy on T1 pN0M0 breast cancer.

\section{Methods}

75,139 patients diagnosed with T1 pNOM0 breast cancer were selected from the Surveillance, Epidemiology, and End Results (SEER) database. Multivariate Cox analyses were performed to investigate the effects of chemotherapy on T1a, $\mathrm{T} 1 \mathrm{~b}$, and T1c pNOMO breast cancer, various tumor grades, and four molecular subtypes. Propensity score matching (PSM) was used to eliminate confounding factors and further verify the results between chemotherapy and no chemotherapy. Finally, 545 T1pN0M0 breast cancer patients treated at the Northern Jiangsu People's Hospital were included for external validation. Univariate and multivariate Cox analyses were used to confirm the role of chemotherapy in T1a, T1b, and T1c pNOM0 breast cancer. Survival curves were plotted using the Kaplan-Meier method for tumor grades and molecular subtypes.

\section{Results}

Chemotherapy demonstrated a statistically significant improvement in T1b and T1c breast cancer, not in T1a breast cancer. With T1b breast cancer, chemotherapy had effects on grade III and molecular subtypes hormone receptor+ [HR+]/human epidermal growth factor receptor 2+ [HER2+], HR-/HER2+, and HR-/HER2-. Chemotherapy was beneficial to overall survival for grade II/III and T1c breast cancer. After PSM, identical results were obtained. We also obtained similar results with external validation, except that chemotherapy made a difference in grade II and T1b breast cancer of external validation.

\section{Conclusion}

Partial T1 pN0M0 breast cancer patients with tumor grade III T1b pN0M0 except HR+/HER2-, those with tumor grade II and III T1c pNOMO can obtain overall survival benefits from chemotherapy.

\section{Introduction}

Breast cancer is the most common female malignancy and the leading cause of cancer-related death worldwide. ${ }^{1}$ The incidence of breast cancer, especially in the early stage, has been increasingly observed in recent decades because of the advancements in and the widespread use of imaging and longer life expectancy. ${ }^{2-5}$ Small tumors without lymph node infiltration have been given much attention by physicians. These cases are considered to have a good prognosis even though patients undergo surgery without adjuvant therapy. T1 pNOMO breast cancer has been shown to have a relatively low risk of death and recurrence. ${ }^{6,7}$ According to the eighth edition of the American Joint Committee on Cancer Staging manual, T1 pN0M0 breast cancer includes tumors smaller than $2 \mathrm{~cm}$ without node involvement. These tumors are subdivided into three groups: T1a $(\leq 0.5 \mathrm{~cm}), \mathrm{T} 1 \mathrm{~b}(>0.5 \mathrm{~cm}$ but $\leq 1.0 \mathrm{~cm})$, and T1c $(>1.0 \mathrm{~cm}$ but $\leq 2.0 \mathrm{~cm}){ }^{8}$

Breast cancer is a heterogeneous disease with distinct therapeutic responses consisting of four various molecular subtypes. ${ }^{9}$ Considering the results of international reviews, adjuvant therapy for breast cancer can reduce deaths by 
approximately $25 \%$ across all risk groups. ${ }^{10}$ Adjuvant systematic therapy includes chemotherapy, radiotherapy, endocrine therapy, and targeted therapy. Although chemotherapy is an effective treatment, it is also toxic to humans and has shortterm and long-term side effects that can result in death. ${ }^{11}$ Patients with early stage breast cancer (EBC) can achieve only a small absolute percentage of survival advantages from chemotherapy. ${ }^{12}$ Therefore, it is crucial to identify which patients with EBC require adjuvant chemotherapy. When considering chemotherapy, the possible risks are compared to the possible benefit. Chemotherapy can not only reduce the risk of recurrence and death but also reduce the damage caused by toxic effects and unnecessary expenses. Nevertheless, without advanced evidence, the possible exclusions from chemotherapy are controversial. Therefore, our study aimed to evaluate the effects of adjuvant chemotherapy on the overall survival of T1 pNOM0 breast cancer patients.

\section{Patients And Methods}

\subsection{Data acquisition and patient selection}

The Surveillance, Epidemiology, and End Results (SEER) database was established in 1973 by the National Cancer Institute and is one of the largest tumor registration databases. Data were selected from 18 registries of the SEER program, which identified T1 pN0M0 female breast cancer patients from 2010 to 2014. Then, exclusion was performed for the following patients: those who did not undergo surgery; those with ductal carcinoma in situ and lobular carcinoma in situ; those who participated in less than 3 months of follow-up; those with unknown data; and those with other primary malignant tumors.

We collected the data of 75,139 EBC patients from the SEER database. During external validation, the same inclusion and exclusion criteria were used for patients treated at Northern Jiangsu People's Hospital from 2010 to 2015 . Finally, 545 female T1 pN0M0 breast cancer patients were included in the study.

Our study was approved by the ethics committee of Northern Jiangsu People's Hospital, Clinical Medical School, Affiliated Hospital of Yangzhou University.

The following data of each patient were gathered: patient number, age, death status (yes/no), follow-up time, tumor size, grade, estrogen receptor (ER) status, progesterone receptor (PR) status, human epidermal growth factor receptor-2 (HER2), surgical method, chemotherapy history, and radiation history. Because chemotherapy-related toxicities can lead to death, we chose overall survival (OS) as the endpoint instead of breast cancer-specific survival. ${ }^{13}$ An age of 60 years was regarded as the threshold for distinguishing young and old patients. ${ }^{14}$

\subsection{SEER methods}

T1 pN0M0 breast cancer patients were divided into three groups: T1a $(n=10,073)$; T1b $(n=24,951)$; and T1c $(n=40,115)$. We conducted a descriptive analysis of the baseline clinical features of eligible patients and used the chi-square test to compare the characteristics of patients among the three groups. The multivariable Cox regression analysis was performed to calculate the effects of chemotherapy on T1a, T1b, and T1c. We also performed a multivariable Cox regression analysis of diverse tumor grades and molecular subtypes. To further confirm the specific role of chemotherapy for those in the three groups and four molecular subtype subgroups, a multivariable Cox regression analysis of tumor grades was performed. Furthermore, propensity score matching (PSM) was performed to balance disparities between chemotherapy and no chemotherapy. The propensity score for chemotherapy status was calculated for each patient using multivariate logistic regression and considered the following imbalanced factors: tumor grade, surgery type, radiation record, molecular subtype, and age. PSM was conducted using a propensity score constructed using 1:1 nearest-neighbor matching within calipers without replacement. ${ }^{15}$ After PSM, to obtain more accurate results, a 
multivariable Cox regression analysis was repeated to assess the usefulness of chemotherapy for the tumor grades and molecular subtypes of the three groups.

\subsection{External validation methods}

EBC patients comprised three groups: T1a $(n=98), T 1 b(n=130)$, and T1c $(n=317)$. Because of a lack of data, we used the univariable Cox regression analysis to identify the significant variables and remove some inconsequential parameters. These meaningful indicators were incorporated in the multivariable Cox regression analysis to further evaluate the three groups. In each of the three groups, Kaplan-Meier survival curves of tumor grades and molecular subtypes were plotted and estimated log-rank test results were used to compare the control group (no chemotherapy) and the experimental group (chemotherapy).

\subsection{Statistical methods}

Data analyses were performed using R software version 3.5.1 (R Foundation for Statistical Computing, Vienna, Austria) and SPSS version 19.0 (IBM Corporation, Armonk, NY, USA). Two-tailed $\mathrm{P}<0.05$ was considered statistically significant.

\section{Results}

\subsection{SEER results}

The median follow-up time was 51 months, as calculated by the reverse Kaplan-Meier method. As shown in Table S1, a cohort of 75,139 eligible patients were identified from the SEER database. T1c NOMO breast cancer patients tended to have worse differentiated tumor grades and more often received adjuvant chemotherapy. There were notable statistical differences among groups $(\mathrm{P}<0.01)$. The outcomes of multivariate Cox proportional hazard analyses of $\mathrm{T} 1, \mathrm{~T} 1 \mathrm{a}, \mathrm{T} 1 \mathrm{~b}$, and T1c are presented in Table S2. Chemotherapy was effective for T1b (hazard ratio [HR], 0.72; 95\% confidence interval [Cl], 0.59-0.88; $\mathrm{P}<0.0001)$ and T1c $(\mathrm{HR}, 0.54 ; 95 \% \mathrm{Cl}, 0.48-0.60 ; \mathrm{P}<0.0001)$. Compared with other subtypes, HR+/HER2had a better prognosis with T1b and T1c. Patients who underwent radiotherapy had more favorable survival rates $(\mathrm{P}<$ 0.0001). Not surprisingly, larger tumors were strong predictors of worse survival. The multivariable Cox regression analysis of four molecular subtypes and tumor grades of T1a are presented in Tables S3 and S4. Chemotherapy did not have a role in molecular subtypes HR+/HER2- $(P=0.11)$, HR+/HER2+ $(P=0.36)$, HR-/HER2+ $(P=0.22)$, and HR-/HER2- $(P$ $=0.20)$ and tumor grades I $(P=0.78)$, II $(P=0.23)$, and III $(P=0.68)$. The multivariable Cox regression analysis of four molecular subtypes and tumor grades of T1b are presented in Tables S5 and S6. Patients with HR+/HER2- $(P=0.67)$, grade II $(P=0.41)$, and grade III $(P=0.11)$ administered chemotherapy received no survival advantages. Patients with $\mathrm{HR}+/ \mathrm{HER} 2+(\mathrm{HR}, 0.41 ; 95 \% \mathrm{Cl}, 0.25-0.69 ; \mathrm{P}<0.0001), \mathrm{HR}-/ \mathrm{HER} 2+(\mathrm{HR}, 0.50 ; 95 \% \mathrm{Cl}, 0.25-1.02 ; \mathrm{P}=0.01), \mathrm{HR}-/ \mathrm{HER} 2-(\mathrm{HR}$, 0.53; 95\% Cl, 0.36-0.78; $\mathrm{P}<0.0001)$, and grade III (HR, 0.52; 95\% $\mathrm{Cl}, 0.38-0.70 ; \mathrm{P}<0.0001)$ who were administered chemotherapy had survival advantages compared with patients who were not. T1c patients who received chemotherapy appeared to obtain OS benefits compared with their counterparts, except for those with grade I $(P=0.10)($ Tables S7 and S8). Multivariable Cox regression analyses of tumor grades of the T1a group and molecular subtype subgroups are presented in Tables S9, S10, S11, and S12. Not surprisingly, chemotherapy did not improve the OS of T1a patients. However, chemotherapy decreased survival time for grade II (HR, 3.23; 95\% Cl, 1.81-5.78; $\mathrm{P}<0.0001)$ and grade III (HR, 3.26; $95 \% \mathrm{Cl}, 1.25-8.48 ; \mathrm{P}=0.02) \mathrm{T} 1$ a patients with subtype HR+/HER2-. Chemotherapy led to accelerated death for grade I T1b patients with HR+/HER2- (HR, 1.62; 95\% Cl, 1.02-2.57; P = 0.04) (Tables S13, S14, S15, and S16). Chemotherapy also significantly enhanced the survival of grade III (HR, $0.36 ; 95 \% \mathrm{Cl}, 0.16-0.80 ; \mathrm{P}=0.01) \mathrm{T} 1 \mathrm{~b}$ patients with HR+/HER2+, HR-/HER2+ (HR, 0.34; 95\% Cl, 0.14-0.84; P= 0.02), and HR-/HER2- (HR, 0.54; 95\% Cl, 0.34-0.85; P = 0.01). However, chemotherapy was not useful for grade I T1c patients with HR+/HER2- $(P=0.20), H R+/ H E R 2+(P=0.28)$, HR-/HER2+ ( $\mathrm{P}=0.41)$, and HR-/HER2- $(\mathrm{P}=0.67)$ (Tables S17, S18, S19, and S20). Tables S21, S22, and S23 summarize the association of chemotherapy with other variables. The results of the chemotherapy group were clearly at odds with 
those of the no chemotherapy group of the entire cohort $(P<0.01)$. After PSM, the parameters of these two groups were similar in the matched cohort. Consequently, these matched data were used for further analyses and validation. In the matched cohort, chemotherapy was statistically significant for T1b $(H R, 0.44 ; 95 \% \mathrm{Cl}, 0.38-0.51 ; \mathrm{P}<0.001)$ and T1c (HR, 0.47; 95\% Cl, 0.41-0.54; $\mathrm{P}<0.001$ ) (Table S24). Similarly, T1a patients of the matched cohort (Tables 1 and 2) with tumor grades I $(P=0.27)$, II $(P=0.99)$, and III $(P=0.57)$ and molecular subtypes HR+/HER2- $(P=0.66), H R+/ H E R 2+(P=$ $0.20)$, HR/HER2+ $(P=0.27)$, and HR-/HER2- $(P=0.48)$ were not sensitive to chemotherapy. Furthermore, T1b patients with HR+/HER2+ (HR, 0.38; 95\% Cl, 0.21-0.66; P<0.01), HR-/HER2+ (HR, 0.51; 95\% Cl, 0.23-1.15; P = 0.01), HR-/HER2- (HR, $0.44 ; 95 \% \mathrm{Cl}, 0.28-0.67 ; \mathrm{P}<0.0001)$ and grade III $(\mathrm{HR}, 0.51 ; 95 \% \mathrm{Cl}, 0.36-0.72 ; \mathrm{P}<0.0001)$ administered chemotherapy received OS benefits (Tables 3 and 4). T1c patients who received chemotherapy appeared to acquire OS benefits compared with their counterparts, except for those with grade I $(P=0.33)$ of the matched cohort (Tables 5 and 6$)$.

Table 1

Multivariable Cox regression analyses of four molecular subtypes of T1a in the matched cohort.

\begin{tabular}{|c|c|c|c|c|c|c|c|c|}
\hline \multirow[t]{3}{*}{ Variable } & \multicolumn{2}{|l|}{ HR+/HER2- } & \multicolumn{2}{|l|}{ HR+/HER2+ } & \multicolumn{2}{|l|}{ HR-/HER2+ } & \multicolumn{2}{|l|}{ HR-/HER2- } \\
\hline & \multicolumn{2}{|c|}{ Multivariate Analysis } & \multicolumn{2}{|c|}{ Multivariate Analysis } & \multicolumn{2}{|l|}{$\begin{array}{l}\text { Multivariate } \\
\text { Analysis }\end{array}$} & \multicolumn{2}{|l|}{$\begin{array}{l}\text { Multivariate } \\
\text { Analysis }\end{array}$} \\
\hline & $\mathrm{HR}(95 \% \mathrm{Cl})$ & $\begin{array}{l}\mathrm{P}- \\
\text { value }\end{array}$ & $\mathrm{HR}(95 \% \mathrm{Cl})$ & $\begin{array}{l}\mathrm{P}- \\
\text { value }\end{array}$ & $\mathrm{HR}(95 \% \mathrm{Cl})$ & $\begin{array}{l}P \text { - } \\
\text { value }\end{array}$ & $\begin{array}{l}\text { HR } \\
(95 \% \mathrm{Cl})\end{array}$ & $\begin{array}{l}P \text { - } \\
\text { value }\end{array}$ \\
\hline \multicolumn{9}{|l|}{ GRADE } \\
\hline I & reference & & reference & & reference & & reference & \\
\hline II & $\begin{array}{l}2.21(0.91- \\
5.36)\end{array}$ & 0.08 & - & - & $\begin{array}{l}0.55(0.07- \\
4.62)\end{array}$ & 0.58 & - & - \\
\hline III & $\begin{array}{l}2.92(1.08- \\
7.92)\end{array}$ & 0.04 & - & - & $\begin{array}{l}0.35(0.04- \\
3.24)\end{array}$ & 0.35 & - & - \\
\hline \multicolumn{9}{|l|}{ SURGERY } \\
\hline Breast-conserving & reference & & reference & & reference & & reference & \\
\hline Total mastectomy & $\begin{array}{l}0.46(0.17- \\
1.23)\end{array}$ & 0.12 & $\begin{array}{l}22.06(2.09- \\
232.47)\end{array}$ & 0.01 & $\begin{array}{l}1.79(0.11- \\
28.43)\end{array}$ & 0.68 & $\begin{array}{l}0.44(0.11- \\
1.85)\end{array}$ & 0.26 \\
\hline $\begin{array}{l}\text { Modified radical } \\
\text { mastectomy }\end{array}$ & $\begin{array}{l}0.29(0.07- \\
1.24)\end{array}$ & 0.09 & - & - & $\begin{array}{l}1.09(0.04- \\
27.13)\end{array}$ & 0.96 & - & - \\
\hline \multicolumn{9}{|l|}{ RADIATION } \\
\hline No & reference & & reference & & reference & & reference & \\
\hline Yes & $\begin{array}{l}0.20(0.07- \\
0.58)\end{array}$ & $<0.01$ & $\begin{array}{l}6.33(0.66- \\
60.72)\end{array}$ & 0.11 & $\begin{array}{l}1.41(0.09- \\
21.27)\end{array}$ & 0.80 & $\begin{array}{l}0.19(0.04- \\
0.85)\end{array}$ & 0.03 \\
\hline \multicolumn{9}{|l|}{ CHEMOTHERAPY } \\
\hline No & reference & & reference & & reference & & reference & \\
\hline Yes & $\begin{array}{l}2.25(1.09- \\
4.62)\end{array}$ & 0.66 & $\begin{array}{l}0.27(0.06- \\
1.25)\end{array}$ & 0.10 & $\begin{array}{l}0.51(0.15- \\
1.70)\end{array}$ & 0.27 & $\begin{array}{l}0.66(0.21- \\
2.10)\end{array}$ & 0.48 \\
\hline \multicolumn{9}{|l|}{ AGE (year) } \\
\hline$\nabla 60$ & reference & & reference & & reference & & reference & \\
\hline$₫ 60$ & $\begin{array}{l}5.22(2.40- \\
11.32)\end{array}$ & $\begin{array}{l}<.0001 \\
0\end{array}$ & $\begin{array}{l}52.09(6.10- \\
444.61)\end{array}$ & $\begin{array}{l}< \\
0.01\end{array}$ & $\begin{array}{l}1.31(0.37- \\
4.67)\end{array}$ & 0.68 & $\begin{array}{l}0.47(0.13- \\
1.77)\end{array}$ & 0.27 \\
\hline
\end{tabular}


Table 2

Multivariable Cox regression analysis of tumor grades of T1a in the matched cohort.

\begin{tabular}{|c|c|c|c|c|c|c|}
\hline \multirow[t]{3}{*}{ Variable } & \multicolumn{2}{|l|}{ T1a: GRADEX } & \multicolumn{2}{|c|}{ T1a: GRADEX } & \multicolumn{2}{|c|}{ T1a: GRADEX } \\
\hline & \multicolumn{2}{|c|}{ Multivariate Analysis } & \multicolumn{2}{|c|}{ Multivariate Analysis } & \multicolumn{2}{|c|}{ Multivariate Analysis } \\
\hline & $\mathrm{HR}(95 \% \mathrm{Cl})$ & $\begin{array}{l}\mathrm{P} \text { - } \\
\text { value }\end{array}$ & $\mathrm{HR}(95 \% \mathrm{Cl})$ & $\begin{array}{l}\mathrm{P}- \\
\text { value }\end{array}$ & $\mathrm{HR}(95 \% \mathrm{Cl})$ & $\begin{array}{l}\mathrm{P} \text { - } \\
\text { value }\end{array}$ \\
\hline \multicolumn{7}{|l|}{ SURGERY } \\
\hline Breast-conserving & reference & & reference & & reference & \\
\hline Total mastectomy & $0.36(0.03-3.94)$ & 0.41 & $\begin{array}{l}2.13(0.59- \\
7.72)\end{array}$ & 0.25 & $\begin{array}{l}0.52(0.14- \\
1.90)\end{array}$ & 0.32 \\
\hline $\begin{array}{l}\text { Modified radical } \\
\text { mastectomy }\end{array}$ & $\begin{array}{l}0.54(0.03- \\
10.37)\end{array}$ & 0.68 & $\begin{array}{l}1.99(0.46- \\
8.65)\end{array}$ & 0.36 & $\begin{array}{l}0.29(0.05- \\
1.78)\end{array}$ & 0.18 \\
\hline \multicolumn{7}{|l|}{ RADIATION } \\
\hline No & reference & & reference & & reference & \\
\hline Yes & - & - & $\begin{array}{l}1.78(0.52- \\
6.12)\end{array}$ & 0.36 & $\begin{array}{l}0.23(0.06- \\
0.87)\end{array}$ & 0.03 \\
\hline \multicolumn{7}{|l|}{ CHEMOTHERAPY } \\
\hline No & reference & & reference & & reference & \\
\hline Yes & $\begin{array}{l}2.54(0.49- \\
13.09)\end{array}$ & 0.27 & $\begin{array}{l}1.00(0.51- \\
1.94)\end{array}$ & 0.99 & $\begin{array}{l}1.27(0.56- \\
2.91)\end{array}$ & 0.57 \\
\hline \multicolumn{7}{|l|}{ SUBTYPE } \\
\hline HR+/HER2- & reference & & reference & & reference & \\
\hline $\mathrm{HR}+/ \mathrm{HER} 2+$ & - & - & $\begin{array}{l}0.22(0.06- \\
0.75)\end{array}$ & 0.02 & $\begin{array}{l}0.10(0.01- \\
0.79)\end{array}$ & 0.03 \\
\hline HR-/HER2+ & $1.73(0.20-15.15)$ & 0.62 & $\begin{array}{l}1.00(0.43- \\
2.33)\end{array}$ & 1.00 & $\begin{array}{l}0.68(0.22- \\
2.15)\end{array}$ & 0.52 \\
\hline HR-/HER2- & - & - & $\begin{array}{l}1.13(0.44- \\
2.93)\end{array}$ & 0.80 & $\begin{array}{l}0.58(0.21- \\
1.57)\end{array}$ & 0.28 \\
\hline \multicolumn{7}{|l|}{ AGE (year) } \\
\hline$₫ 60$ & reference & & reference & & reference & \\
\hline$₫ 60$ & $0.31(0.03-3.20)$ & 0.32 & $\begin{array}{l}3.35(1.64- \\
6.83)\end{array}$ & $<0.01$ & $\begin{array}{l}2.89(1.25- \\
6.68)\end{array}$ & 0.01 \\
\hline
\end{tabular}


Table 3

Multivariable Cox regression analyses of four molecular subtypes of T1b in the matched cohort

\begin{tabular}{|c|c|c|c|c|c|c|c|c|}
\hline \multirow[t]{3}{*}{ Variable } & \multicolumn{2}{|l|}{ HR+/HER2- } & \multicolumn{2}{|l|}{ HR+/HER2+ } & \multicolumn{2}{|l|}{ HR-/HER2+ } & \multicolumn{2}{|l|}{ HR-/HER2- } \\
\hline & \multicolumn{2}{|c|}{ Multivariate Analysis } & \multicolumn{2}{|c|}{ Multivariate Analysis } & \multicolumn{2}{|l|}{$\begin{array}{l}\text { Multivariate } \\
\text { Analysis }\end{array}$} & \multicolumn{2}{|c|}{ Multivariate Analysis } \\
\hline & $\begin{array}{l}\text { HR } \\
(95 \% \mathrm{Cl})\end{array}$ & $\begin{array}{l}\mathrm{P} \text { - } \\
\text { value }\end{array}$ & $\mathrm{HR}(95 \% \mathrm{Cl})$ & $\begin{array}{l}\mathrm{P}- \\
\text { value }\end{array}$ & $\begin{array}{l}\mathrm{HR} \\
(95 \% \mathrm{Cl})\end{array}$ & $\begin{array}{l}\mathrm{P}- \\
\text { value }\end{array}$ & $\begin{array}{l}\mathrm{HR} \\
(95 \% \mathrm{Cl})\end{array}$ & $\begin{array}{l}P \text { - } \\
\text { value }\end{array}$ \\
\hline \multicolumn{9}{|l|}{ GRADE } \\
\hline I & reference & & reference & & reference & & reference & \\
\hline II & $\begin{array}{l}0.82(0.52- \\
1.29)\end{array}$ & 0.39 & $\begin{array}{l}0.74(0.30- \\
1.85)\end{array}$ & 0.52 & $\begin{array}{l}0.83(0.10- \\
6.79)\end{array}$ & 0.87 & $\begin{array}{l}1.29(0.31- \\
5.44)\end{array}$ & 0.73 \\
\hline III & $\begin{array}{l}0.93(0.58- \\
1.48)\end{array}$ & 0.76 & $\begin{array}{l}0.88(0.35- \\
2.23)\end{array}$ & 0.79 & $\begin{array}{l}0.87(0.11- \\
6.77)\end{array}$ & 0.89 & $\begin{array}{l}1.32(0.32- \\
5.42)\end{array}$ & 0.70 \\
\hline \multicolumn{9}{|l|}{ SURGERY } \\
\hline Breast-conserving & reference & & reference & & reference & & reference & \\
\hline Total mastectomy & $\begin{array}{l}1.14(0.61- \\
2.11)\end{array}$ & 0.68 & $\begin{array}{l}0.64(0.28- \\
1.46)\end{array}$ & 0.29 & $\begin{array}{l}0.28(0.09- \\
0.84)\end{array}$ & 0.02 & $\begin{array}{l}1.65(0.77- \\
3.54)\end{array}$ & 0.20 \\
\hline $\begin{array}{l}\text { Modified radical } \\
\text { mastectomy }\end{array}$ & $\begin{array}{l}1.64(0.81- \\
3.30)\end{array}$ & 0.17 & $\begin{array}{l}1.05(0.34- \\
3.23)\end{array}$ & 0.94 & $\begin{array}{l}0.40(0.09- \\
1.70)\end{array}$ & 0.21 & $\begin{array}{l}1.79(0.72- \\
4.44)\end{array}$ & 0.21 \\
\hline \multicolumn{9}{|l|}{ RADIATION } \\
\hline No & reference & & reference & & reference & & reference & \\
\hline Yes & $\begin{array}{l}0.63(0.35- \\
1.14)\end{array}$ & 0.13 & $\begin{array}{l}0.59(0.27- \\
1.27)\end{array}$ & 0.18 & $\begin{array}{l}0.33(0.10- \\
1.06)\end{array}$ & 0.06 & $\begin{array}{l}0.93(0.45- \\
1.93)\end{array}$ & 0.84 \\
\hline \multicolumn{9}{|l|}{ CHEMOTHERAPY } \\
\hline No & reference & & reference & & reference & & reference & \\
\hline Yes & $\begin{array}{l}0.94(0.66- \\
1.34)\end{array}$ & 0.74 & $\begin{array}{l}0.38(0.21- \\
0.66)\end{array}$ & $<0.01$ & $\begin{array}{l}0.51(0.23- \\
1.15)\end{array}$ & 0.01 & $\begin{array}{l}0.44(0.28- \\
0.67)\end{array}$ & $\begin{array}{l}< \\
0.0001\end{array}$ \\
\hline \multicolumn{9}{|l|}{ AGE (year) } \\
\hline$\nabla 60$ & reference & & reference & & reference & & reference & \\
\hline$₫ 60$ & $\begin{array}{l}3.92(2.67- \\
5.74)\end{array}$ & $<0.0001$ & $\begin{array}{l}4.93(2.37- \\
10.28)\end{array}$ & $\begin{array}{l}< \\
0.0001\end{array}$ & $\begin{array}{l}2.34(0.87- \\
6.32)\end{array}$ & 0.09 & $\begin{array}{l}3.34(1.81- \\
6.15)\end{array}$ & $\begin{array}{l}<.0001 \\
0.001\end{array}$ \\
\hline
\end{tabular}


Table 4

Multivariable Cox regression analyses of tumor grades of $\mathrm{T} 1 \mathrm{~b}$ in the matched cohort.

\begin{tabular}{|c|c|c|c|c|c|c|}
\hline \multirow[t]{3}{*}{ Variable } & \multicolumn{2}{|c|}{ T1b: GRADEX } & \multicolumn{2}{|c|}{ T1b: GRADEX } & \multicolumn{2}{|c|}{ T1b: GRADEX } \\
\hline & \multicolumn{2}{|c|}{ Multivariate Analysis } & \multicolumn{2}{|c|}{ Multivariate Analysis } & \multicolumn{2}{|c|}{ Multivariate Analysis } \\
\hline & $\mathrm{HR}(95 \% \mathrm{Cl})$ & P-value & $\mathrm{HR}(95 \% \mathrm{Cl})$ & P-value & $\mathrm{HR}(95 \% \mathrm{Cl})$ & P-value \\
\hline \multicolumn{7}{|l|}{ SURGERY } \\
\hline Breast-conserving & reference & & reference & & reference & \\
\hline Total mastectomy & $\begin{array}{l}0.88(0.35- \\
2.19)\end{array}$ & 0.78 & $\begin{array}{l}1.23(0.60- \\
2.52)\end{array}$ & 0.58 & $\begin{array}{l}0.91(0.50- \\
1.66)\end{array}$ & 0.77 \\
\hline $\begin{array}{l}\text { Modified radical } \\
\text { mastectomy }\end{array}$ & $\begin{array}{l}2.34(0.79- \\
6.96)\end{array}$ & 0.13 & $\begin{array}{l}2.06(0.91- \\
4.67)\end{array}$ & 0.08 & $\begin{array}{l}0.71(0.32- \\
1.58)\end{array}$ & 0.40 \\
\hline \multicolumn{7}{|l|}{ RADIATION } \\
\hline No & reference & & reference & & reference & \\
\hline Yes & $\begin{array}{l}0.55(0.23- \\
1.34)\end{array}$ & 0.19 & $\begin{array}{l}0.83(0.41- \\
1.65)\end{array}$ & 0.59 & $\begin{array}{l}0.76(0.43- \\
1.36)\end{array}$ & 0.36 \\
\hline \multicolumn{7}{|l|}{ CHEMOTHERAPY } \\
\hline No & reference & & reference & & reference & \\
\hline Yes & $\begin{array}{l}0.83(0.45- \\
1.52)\end{array}$ & 0.54 & $\begin{array}{l}0.80(0.55- \\
1.18)\end{array}$ & 0.26 & $\begin{array}{l}0.51(0.36- \\
0.72)\end{array}$ & $<.0001$ \\
\hline \multicolumn{7}{|l|}{ SUBTYPE } \\
\hline HR+/HER2- & reference & & reference & & reference & \\
\hline HR+/HER2+ & $\begin{array}{l}1.68(0.77- \\
3.64)\end{array}$ & 0.19 & $\begin{array}{l}1.10(0.68- \\
1.78)\end{array}$ & 0.70 & $\begin{array}{l}1.25(0.74- \\
2.10)\end{array}$ & 0.41 \\
\hline HR-/HER2+ & $\begin{array}{l}1.13(0.15- \\
8.40)\end{array}$ & 0.90 & $\begin{array}{l}1.99(0.96- \\
4.13)\end{array}$ & 0.06 & $\begin{array}{l}2.09(1.19- \\
3.68)\end{array}$ & 0.01 \\
\hline HR-/HER2- & - & - & $\begin{array}{l}1.32(0.78- \\
2.23)\end{array}$ & 0.31 & $\begin{array}{l}1.47(0.97- \\
2.23)\end{array}$ & 0.07 \\
\hline \multicolumn{7}{|l|}{ AGE (year) } \\
\hline$\bigotimes 60$ & reference & & reference & & reference & \\
\hline$\triangle 60$ & $\begin{array}{l}\text { 6.42(3.13- } \\
13.17)\end{array}$ & $\begin{array}{l}<.0001 \\
0 .\end{array}$ & $\begin{array}{l}2.70(1.74- \\
4.20)\end{array}$ & $\begin{array}{l}<.0001 \\
0.00\end{array}$ & $\begin{array}{l}2.44(1.62- \\
3.67)\end{array}$ & $\begin{array}{l}<.0001 \\
0 .\end{array}$ \\
\hline
\end{tabular}


Table 5

Multivariable Cox regression analyses of four molecular subtypes of T1c in the matched cohort.

\begin{tabular}{|c|c|c|c|c|c|c|c|c|}
\hline \multirow[t]{3}{*}{ Variable } & \multirow{2}{*}{\multicolumn{2}{|c|}{$\begin{array}{l}\text { HR+/HER2- } \\
\text { Multivariate Analysis }\end{array}$}} & \multirow{2}{*}{\multicolumn{2}{|c|}{$\begin{array}{l}\text { HR+/HER2+ } \\
\text { Multivariate Analysis }\end{array}$}} & \multirow{2}{*}{\multicolumn{2}{|c|}{$\begin{array}{l}\text { HR-/HER2+ } \\
\text { Multivariate Analysis }\end{array}$}} & \multirow{2}{*}{\multicolumn{2}{|c|}{$\begin{array}{l}\text { HR-/HER2- } \\
\text { Multivariate Analysis }\end{array}$}} \\
\hline & & & & & & & & \\
\hline & $\begin{array}{l}\mathrm{HR} \\
(95 \% \mathrm{Cl})\end{array}$ & $\begin{array}{l}\mathrm{P}- \\
\text { value }\end{array}$ & $\begin{array}{l}\mathrm{HR} \\
(95 \% \mathrm{Cl})\end{array}$ & $\begin{array}{l}\mathrm{P}- \\
\text { value }\end{array}$ & $\begin{array}{l}\mathrm{HR} \\
(95 \% \mathrm{Cl})\end{array}$ & $\begin{array}{l}\mathrm{P}- \\
\text { value }\end{array}$ & $\begin{array}{l}\mathrm{HR} \\
(95 \% \mathrm{Cl})\end{array}$ & $\begin{array}{l}P \text { - } \\
\text { value }\end{array}$ \\
\hline \multicolumn{9}{|l|}{ GRADE } \\
\hline I & reference & & reference & & reference & & reference & \\
\hline II & $\begin{array}{l}1.27(0.92- \\
1.77)\end{array}$ & 0.14 & $\begin{array}{l}1.02(0.54- \\
1.93)\end{array}$ & 0.95 & $\begin{array}{l}0.19(0.04- \\
0.88)\end{array}$ & 0.03 & $\begin{array}{l}1.71(0.69- \\
4.24)\end{array}$ & 0.25 \\
\hline III & $\begin{array}{l}1.74(1.26- \\
2.40)\end{array}$ & $<0.01$ & $\begin{array}{l}1.12(0.58- \\
2.13)\end{array}$ & 0.74 & $\begin{array}{l}0.19(0.04- \\
0.83)\end{array}$ & 0.03 & $\begin{array}{l}2.19(0.90- \\
5.31)\end{array}$ & 0.08 \\
\hline \multicolumn{9}{|l|}{ SURGERY } \\
\hline $\begin{array}{l}\text { Breast- } \\
\text { conserving }\end{array}$ & reference & & reference & & reference & & reference & \\
\hline $\begin{array}{l}\text { Total } \\
\text { mastectomy }\end{array}$ & $\begin{array}{l}0.54(0.40- \\
0.74)\end{array}$ & $<.0001$ & $\begin{array}{l}0.60(0.40- \\
0.89)\end{array}$ & 0.01 & $\begin{array}{l}0.41(0.21- \\
0.78)\end{array}$ & 0.01 & $\begin{array}{l}0.67(0.49- \\
0.91)\end{array}$ & 0.01 \\
\hline $\begin{array}{l}\text { Modified radical } \\
\text { mastectomy }\end{array}$ & $\begin{array}{l}0.75(0.52- \\
1.09)\end{array}$ & 0.13 & $\begin{array}{l}0.52(0.29- \\
0.93)\end{array}$ & 0.03 & $\begin{array}{l}0.53(0.23- \\
1.21)\end{array}$ & 0.13 & $\begin{array}{l}0.55(0.36- \\
0.85)\end{array}$ & 0.01 \\
\hline \multicolumn{9}{|l|}{ RADIATION } \\
\hline No & reference & & reference & & reference & & reference & \\
\hline Yes & $\begin{array}{l}0.36(0.26- \\
0.48)\end{array}$ & $\begin{array}{l}< \\
0.0001\end{array}$ & $\begin{array}{l}0.37(0.25- \\
0.56)\end{array}$ & $\begin{array}{l}< \\
0.0001\end{array}$ & $\begin{array}{l}0.36(0.16- \\
0.77)\end{array}$ & 0.01 & $\begin{array}{l}0.48(0.35- \\
0.66)\end{array}$ & $\begin{array}{l}<.0001 \\
0.001\end{array}$ \\
\hline \multicolumn{9}{|l|}{ CHEMOTHERAPY } \\
\hline No & reference & & reference & & reference & & reference & \\
\hline Yes & $\begin{array}{l}0.61(0.51- \\
0.74)\end{array}$ & $\begin{array}{l}< \\
0.0001\end{array}$ & $\begin{array}{l}0.42(0.30- \\
0.58)\end{array}$ & < 0.0001 & $\begin{array}{l}0.33(0.19- \\
0.59)\end{array}$ & $\begin{array}{l}< \\
0.0001\end{array}$ & $\begin{array}{l}0.35(0.27- \\
0.44)\end{array}$ & $\begin{array}{l}< \\
0.0001\end{array}$ \\
\hline \multicolumn{9}{|l|}{ AGE (year) } \\
\hline$₫ 60$ & reference & & reference & & reference & & reference & \\
\hline$₫ 60$ & $\begin{array}{l}3.62(2.98- \\
4.41)\end{array}$ & $\begin{array}{l}<.0001 \\
0.001\end{array}$ & $\begin{array}{l}3.27(2.13- \\
5.02)\end{array}$ & $\begin{array}{l}<.0001 \\
0.01\end{array}$ & $\begin{array}{l}2.59(1.34- \\
5.04)\end{array}$ & $<0.01$ & $\begin{array}{l}2.01(1.50- \\
2.69)\end{array}$ & $\begin{array}{l}< \\
0.0001\end{array}$ \\
\hline
\end{tabular}


Table 6

Multivariable Cox regression analyses of tumor grades of T1c in the matched cohort.

\begin{tabular}{|c|c|c|c|c|c|c|}
\hline \multirow[t]{3}{*}{ Variable } & \multicolumn{2}{|c|}{ T1c: GRADEX } & \multicolumn{2}{|c|}{ T1c: GRADEX } & \multicolumn{2}{|c|}{ T1c: GRADEX } \\
\hline & \multicolumn{2}{|c|}{ Multivariate Analysis } & \multicolumn{2}{|c|}{ Multivariate Analysis } & \multicolumn{2}{|c|}{ Multivariate Analysis } \\
\hline & $\mathrm{HR}(95 \% \mathrm{Cl})$ & P-value & $\mathrm{HR}(95 \% \mathrm{Cl})$ & P-value & $\mathrm{HR}(95 \% \mathrm{Cl})$ & P-value \\
\hline \multicolumn{7}{|l|}{ SURGERY } \\
\hline Breast-conserving & reference & & reference & & reference & \\
\hline Total mastectomy & $\begin{array}{l}0.63(0.26- \\
1.52)\end{array}$ & 0.31 & $\begin{array}{l}0.51(0.37- \\
0.71)\end{array}$ & $\begin{array}{l}< \\
0.0001\end{array}$ & $\begin{array}{l}0.63(0.49- \\
0.80)\end{array}$ & $<0.01$ \\
\hline $\begin{array}{l}\text { Modified radical } \\
\text { mastectomy }\end{array}$ & $\begin{array}{l}1.04(0.39- \\
2.78)\end{array}$ & 0.94 & $\begin{array}{l}0.60(0.39- \\
0.93)\end{array}$ & 0.02 & $\begin{array}{l}0.57(0.42- \\
0.79)\end{array}$ & $<0.01$ \\
\hline \multicolumn{7}{|l|}{ RADIATION } \\
\hline No & reference & & reference & & reference & \\
\hline Yes & $\begin{array}{l}0.43(0.18- \\
1.01)\end{array}$ & 0.05 & $\begin{array}{l}0.40(0.29- \\
0.55)\end{array}$ & $<.0001$ & $\begin{array}{l}0.43(0.34- \\
0.55)\end{array}$ & $<.0001$ \\
\hline \multicolumn{7}{|l|}{ CHEMOTHERAPY } \\
\hline No & reference & & reference & & reference & \\
\hline Yes & $\begin{array}{l}0.78(0.48- \\
1.28)\end{array}$ & 0.33 & $\begin{array}{l}0.53(0.43- \\
0.66)\end{array}$ & $<.0001$ & $\begin{array}{l}0.44(0.37- \\
0.53)\end{array}$ & $<.0001$ \\
\hline \multicolumn{7}{|l|}{ SUBTYPE } \\
\hline HR+/HER2- & reference & & reference & & reference & \\
\hline HR+/HER2+ & $\begin{array}{l}1.70(0.92- \\
3.14)\end{array}$ & 0.09 & $\begin{array}{l}1.53(1.17- \\
2.01)\end{array}$ & $<0.01$ & $\begin{array}{l}1.26(0.96- \\
1.65)\end{array}$ & 0.09 \\
\hline HR-/HER2+ & $\begin{array}{l}6.54(0.88- \\
48.72)\end{array}$ & 0.07 & $\begin{array}{l}2.16(1.30- \\
3.59)\end{array}$ & $<0.01$ & $\begin{array}{l}1.14(0.79- \\
1.63)\end{array}$ & 0.49 \\
\hline HR-/HER2- & $\begin{array}{l}1.58(0.61- \\
4.07)\end{array}$ & 0.34 & $\begin{array}{l}2.05(1.53- \\
2.73)\end{array}$ & $\begin{array}{l}< \\
0.0001\end{array}$ & $\begin{array}{l}1.81(1.50- \\
2.20)\end{array}$ & $<.0001$ \\
\hline \multicolumn{7}{|l|}{ AGE (year) } \\
\hline$\varangle 60$ & reference & & reference & & reference & \\
\hline$\$ 60$ & $\begin{array}{l}3.38(1.98- \\
5.77)\end{array}$ & $\begin{array}{l}<.0001 \\
0 .\end{array}$ & $\begin{array}{l}3.68(2.82- \\
4.81)\end{array}$ & $\begin{array}{l}< \\
0.0001\end{array}$ & $\begin{array}{l}2.19(1.81- \\
2.66)\end{array}$ & $\begin{array}{l}<.0001 \\
0\end{array}$ \\
\hline
\end{tabular}

\subsection{External validation results}

The median follow-up time was 60 months, as calculated by the reverse Kaplan-Meier method. A total of 545 T1N0M0 female breast cancer patients were included from Northern Jiangsu People's Hospital (Table S25). Larger tumors tended to have worse differentiated tumor grades and more often received chemotherapy $(P<0.01)$. Results of the multivariable and univariable Cox regression analyses of T1a, T1b, and T1c are presented in Tables S26, S27, and S28. T1a EBC patients did not benefit from adjuvant chemotherapy $(\mathrm{P}=0.47)$. Nevertheless, patients with $\mathrm{T} 1 \mathrm{~b}(\mathrm{HR}, 0.02 ; 95 \% \mathrm{Cl}, 0.00-$ $0.09 ; \mathrm{P}<0.0001)$ and T1c $(\mathrm{HR}, 0.06 ; 95 \% \mathrm{Cl}, 0.03-0.11 ; \mathrm{P}<0.0001)$ breast cancer had prolonged survival. The KaplanMeier survival curves are plotted and include survival curves of tumor grades and molecular subtypes for $T 1 a, T 1 b$, and T1c. For T1a breast cancer patients (Figure S1), chemotherapy was ineffective for those with grade I $(P=0.43)$, grade II 
$(p=0.25), H R+/ H E R 2-(P=0.75), H R+/ H E R 2+(P=0.26)$, and HR-/HER2+ $(P=1)$. Grade III and triple-negative breast cancer could not be plotted because of limited data for T1a. For patients with grade II $(P<0.0001)$, grade III $(P<0.01)$, HR+/HER2+ $(P<0.01)$, HR-/HER2+ $(P<0.0001)$, and HR/HER2- $(P<0.0001)$ T1b breast cancer (Fig. 1), chemotherapy had beneficial effects on OS. Chemotherapy improved OS $(\mathrm{P}<0.0001)$ for those with T1c breast cancer (Figure S2), but not for those with grade I $(P=0.07)$.

\section{Discussion}

In spite of the dramatic increase in the number of EBC patients, ${ }^{3-5,16}$ chemotherapy for T1 pNOM0 breast cancer remains controversial. Therefore, it is imperative to establish a safe, specific, and effective chemotherapy strategy to guide treatment and improve the prognosis of these patients.

In addition to creating the strategy, we utilized PSM and external validation to verify the association between chemotherapy and significant survival advantages obtained by breast cancer patients with T1 pNOM0.

Chemotherapy is recognized as a primary systematic adjuvant modality; however, it negatively influences survival and reduces the quality of life because of its short-term toxicities, including alopecia, nausea, vomiting, and fatigue, and potential long-term effects, including myelosuppression, cardiovascular toxicity, neurotoxicity, marrow neoplasm, and cessation of menses and fertility. ${ }^{17-20}$ EBC patients are expected to survive their cancer diagnosis; however, it is not appropriate to select breast cancer-specific mortality as an endpoint. Therefore, adjuvant therapy has been crucial for observing improvements in the OS of breast cancer patients. ${ }^{21,22}$ Mortality should be considered because it can be caused by toxicities related to chemotherapy. Furthermore, our study suggests that chemotherapy possibly accelerates death for some HR+/HER2-T1ab pNOM0 patients.

Postmastectomy radiation therapy is widely considered to reduce the risk of local recurrence and breast cancer mortality, especially for patients with locally advanced breast cancer who are at high risk because of large tumors and axillary lymph node involvement. ${ }^{23}$ However, the majority of T1 pNOMO breast cancer patients prefer to undergo breastconserving surgery instead of mastectomy, and adjuvant radiotherapy is an indivisible locoregional treatment for breastconserving surgery that achieves local control benefits and OS advantages. ${ }^{24-29}$ These results are consistent with the results of our study.

There was an obvious contradiction in the results obtained using the SEER database and those of external validation. The external validation results indicated that patients with grade II T1b pNOMO can acquire survival benefit from chemotherapy, but the SEER database results did not. There are two interpretations of this phenomenon. First, the data used for external validation are relatively limited; therefore, inevitable deviations may have occurred during statistical analyses. Second, the two cohorts of data were derived from China and the United States. Admittedly, the factors that affect a patient's lifetime vary from country to country and include cultural barriers, ethnic differences, and genetics. ${ }^{30}$ Consequently, the final conclusions refer to the results obtained using the SEER database.

Our research results, the guidelines of the National Comprehensive Cancer Network (NCCN), and the guidelines of the St. Gallen International Breast Cancer Conference (BCC) are nearly identical. ${ }^{31,32}$ The NCCN suggests the following:

1. For node-negative HR+/HER2- breast cancer, if the tumor is $0.5 \mathrm{~cm}$ or smaller, chemotherapy is not recommended. If the tumor is larger than $0.5 \mathrm{~cm}$, then performing a 21 -gene reverse-transcription polymerase chain reaction assay (Oncotype DX) is strongly considered. ${ }^{33-35}$

a. If the recurrence score is $\geq 31$, the risk of recurrence is high and chemotherapy is recommended. 
b. If the recurrence score is $26-30$, the risk of recurrence is moderate and the decision to perform chemotherapy is based on other clinical factors.

c. If the recurrence score is $<26$, the risk of recurrence is low and chemotherapy is not recommended.

2. For node-negative HR+/HER2 + breast cancer, if the tumor is $1.0 \mathrm{~cm}$ or smaller, it is uncertain whether chemotherapy is required. However, chemotherapy is recommended for T1a category 2B, which means that there is an NCCN consensus that intervention is appropriate based on lower-level evidence. If the tumor is larger than $1.0 \mathrm{~cm}$, chemotherapy is recommended.

3. For node-negative HR-/HER2 + breast cancer, chemotherapy is recommended. However, chemotherapy is recommended for category $2 \mathrm{~B}$ when the tumor is smaller than $0.5 \mathrm{~cm}$.

4. For node-negative HR-/HER2- breast cancer, if the tumor is smaller than $0.5 \mathrm{~cm}$, chemotherapy is not recommended. However, chemotherapy is necessary for any other case.

The BCC guidelines are different but somewhat similar to the NCCN guidelines. ${ }^{32}$ Routine adjuvant chemotherapy is not recommended for T1a pN0 breast cancer; this is identical to our research results. The BCC panel recommends adjuvant chemotherapy for HER2-positive and triple-negative breast cancer (TNBC) stage T1b pN0 and higher. For ER+/HER2T1pN0 breast cancer, regardless of luminal-A-like qualities (strongly ER-positive and PR-positive, HER2-negative, with lower grade and proliferation markers) or luminal-B-like tumors, the BCC panel does not recommend adjuvant chemotherapy for patients with low genomic risk scores according to the Oncotype DX and 70-gene signature tests (MammaPrint). ${ }^{36-38}$ Additionally, the European Society for Medical Oncology guidelines are in agreement with the St. Gallen guidelines regarding chemotherapy for EBC. ${ }^{39}$

In our opinion, which is supported by the St. Gallen guidelines, adjuvant chemotherapy should not be performed for T1a pNO breast cancer.

For HR+/HER2- T1bc pN0 breast cancer, chemotherapy is recommended for grade II and grade III T1c pN0 breast cancer if no genetic signature test has been performed or if the 21-gene assay indicates a medium risk. If the conditions are suitable, then we propose following the guidelines to accomplish genetic testing for these patients. For the other three molecular subtypes, chemotherapy is recommended for stage T1b pN0 and higher; this is also mentioned in the St. Gallen guidelines. Some retrospective studies demonstrated the survival benefits of adjuvant chemotherapy for patients with T1C NOMO TNBC. ${ }^{40,41}$ We incorporated tumor grade, which is an independent prognostic indicator, to assess the effects of chemotherapy. ${ }^{42-44}$ Patients, including those with TNBC, can be exempt from adjuvant chemotherapy if they have grade I/II T1b pN0 and grade I T1c pN0 breast cancer.

There were several limitations to our study. First, the SEER database was devoid of variables for genes and therapies, such as the 21-gene assay and schemes and dosages of chemotherapy and endocrine therapies. Second, because the endpoint was OS, age was a significant factor that could not be included to evaluate the effects of chemotherapy. Third, 
because we used a retrospective cohort population, inevitable selection bias might have affected the conclusions.

Further large-scale, prospective, randomized, controlled trials are warranted to accurately identify the outcomes.

\section{Conclusion}

Our study found that chemotherapy is not useful and may even be detrimental to T1a pNO breast cancer patients. Moreover, chemotherapy is recommended for tumor grade III T1b pNO and grade II/III T1c pNO breast cancer, but not with HR+/HER2-. Regarding the molecular subtype HR+/HER2-, in the absence of genetic testing, adjuvant chemotherapy is suggested for tumor grade II and grade III T1c pNO breast cancer.

\section{Declarations}

\section{Ethics approval and consent to participate}

The SEER Program collects data from population-based cancer registries with anonymous information. The SEER is a publicly available database and data extracted from SEER was deemed "non-human study" by the North Shore LIJ IRB committee.

This article is a retrospective review of patient data, and the study design was approved by the Medical Ethics Committee of Northern Jiangsu People's Hospital. This article does not contain any studies with animals or human participants performed by any of the authors.

For this type of study formal consent is not required, and the need for informed consent was waived by the ethics committee.

\section{Consent for publication}

Yes

\section{Availability of data and material}

Yes

\section{Competing interests}

The authors have no conflict of interest to disclose.

\section{Funding}

The study was granted by National Natural Science Foundation of China (82072909).

\section{Authors' contributions}

The first 3 authors contributed equally to this article. Kaiwen Shen: Writing-original draft and formal analysis. Longdi Yao and Huihua Cao: data acquisition and editing. Ximing Gu and Jie Wang: Writing-review and editing.Jinyuan Zhu and Deyuan Fu: Conceptualization and funding acquisition

\section{Acknowledgements}

The authors thank the data managers and staff of the Surveillance, Epidemiology, and End Results (SEER) database for the collection of data in the USA Cancer Registry. We would like to thank the patients from Northern Jiangsu People's 
Hospital for the clinical data.

\section{References}

1. RL S, KD M. A J: Cancer statistics, 2019. Cancer J Clin. 2019;69(1):7-34.

2. HG W, PC P, AJ OM. BS K: Breast-Cancer Tumor Size, Overdiagnosis, and Mammography Screening Effectiveness. N Engl J Med. 2016;375(15):1438-47.

3. The Full Potential of Breast Cancer Screening Use to Reduce Mortality has not yet been Realized in the United States. Breast Cancer Research \& Treatment 2004, 85(3):219-222.

4. Bhoo-Pathy N, Subramaniam S, Taib NA, Hartman M, Alias Z, Tan GH, Ibrahim RI, Yip CH, Verkooijen HM. Spectrum of very early breast cancer in a setting without organised screening. Br J Cancer. 2014;110(9):2187-94.

5. SF M, CA C, SL DDKHJL, PL H-R NPRGU. DO S et al: Recent breast cancer incidence trends according to hormone therapy use: the California Teachers Study cohort. Breast cancer research: BCR. 2010;12(1):R4.

6. Rosen PR, Groshen S, Saigo PE, Kinne DW, Hellman S. A long-term follow-up study of survival in stage I (T1NOM0) and stage II (T1N1M0) breast carcinoma. Journal of Clinical Oncology Official Journal of the American Society of Clinical Oncology, 7(3):355-366.

7. AK L, RA MLGMSB. D, GJ H, K H: Lymph node negative invasive breast carcinoma 1 centimeter or less in size (T1a,bNOMO): clinicopathologic features and outcome. Cancer. 1997;79(4):761-71.

8. Giuliano AE, Edge SB, Hortobagyi GN. Eighth Edition of the AJCC Cancer Staging Manual: Breast Cancer. Ann Surg Oncol. 2018;25(7):1783-5.

9. CA P. V C: Risk of mortality of node-negative, ER/PR/HER2 breast cancer subtypes in T1, T2, and T3 tumors. Breast cancer research treatment. 2017;165(3):743-50.

10. BF C, RD G. S G, AS C, A G: Polychemotherapy for early breast cancer: an overview of the randomised clinical trials with quality-adjusted survival analysis. Lancet. 2001;358(9278):277-86.

11. JJ T, AC KV. W: Long term side effects of adjuvant chemotherapy in patients with early breast cancer. Breast (Edinburgh, Scotland) 2015:S149-153.

12. PM R, IA S. JA H: Survey of breast cancer patients concerning their knowledge and expectations of adjuvant therapy. Journal of clinical oncology: official journal of the American Society of Clinical Oncology. 1998;16(2):515-21.

13. Feuer EJ. Effect of Screening and Adjuvant Therapy on Mortality from Breast Cancer. The New England journal of medicine, 353(17):1784-1792.

14. Kowal P, Dowd J: Definition of an older person. Proposed working definition of an older person in Africa for the MDS Project; 2001.

15. Austin PC. Optimal caliper widths for propensity-score matching when estimating differences in means and differences in proportions in observational studies. Pharmaceutical Statistics 2011, 10.

16. Kennedy T, Stewart AK, Bilimoria KY, Patel-Parekh L, Sener SF, Winchester DP. Treatment Trends and Factors Associated with Survival in T1aN0 and T1bN0 Breast Cancer Patients. Ann Surg Oncol. 2007;14(10):2918-27.

17. DD VH, MW L, HL PB, AL D, VH MR, FM M. Risk factors for doxorubicin-induced congestive heart failure. Ann Intern Med. 1979;91(5):710-7.

18. Weekly Paclitaxel in the Adjuvant Treatment of Breast Cancer. New England Journal of Medicine 2009, 360(16):1685-1685.

19. RE S. Risk for the development of treatment-related acute myelocytic leukemia and myelodysplastic syndrome among patients with breast cancer: review of the literature and the National Surgical Adjuvant Breast and Bowel Project experience. Clin Breast Cancer. 2003;4(4):273-9. 
20. JA P, MJ N, LD C, ED P, EZ N, SE S. P S: Incidence, time course, and determinants of menstrual bleeding after breast cancer treatment: a prospective study. Journal of clinical oncology: official journal of the American Society of Clinical Oncology. 2006;24(7):1045-51.

21. DA B, KA C, SK P, DG F, JS LCMZ, AY M, JD Y, EJ H. F: Effect of screening and adjuvant therapy on mortality from breast cancer. N Engl J Med. 2005;353(17):1784-92.

22. EO H, AM G-A, SH G, KR RR, GN B. H, V V: Overall survival and cause-specific mortality of patients with stage T1a,bNOM0 breast carcinoma. Journal of clinical oncology: official journal of the American Society of Clinical Oncology. 2007;25(31):4952-60.

23. M G-A, C AMRC R: Postmastectomy radiation therapy in early breast cancer. Utility or futility? Critical reviews in oncology/hematology 2020, 147:102887.

24. Fisher B, Anderson S, Redmond CK, Wolmark N, Wickerham DL, Cronin WM. Reanalysis and results after 12 years of follow-up in a randomized clinical trial comparing total mastectomy with lumpectomy with or without irradiation in the treatment of breast cancer. N Engl J Med. 1995;333(22):1456-61.

25. Liljegren G, Holmberg L, Bergh J, Lindgren A, Tabár L, Nordgren H, Adami HO. 10-Year results after sector resection with or without postoperative radiotherapy for stage I breast cancer: a randomized trial. J Clin Oncol. 1999;17(8):2326-33.

26. Fisher B, Bryant J, Dignam JJ, Wickerham DL, Mamounas EP, Fisher ER, Margolese RG, Nesbitt L, Paik S, Pisansky $\mathrm{TM}$, et al. Tamoxifen, radiation therapy, or both for prevention of ipsilateral breast tumor recurrence after lumpectomy in women with invasive breast cancers of one centimeter or less. J Clin Oncol. 2002;20(20):4141-9.

27. Winzer KJ, Sauerbrei W, Braun M, Liersch T, Dunst J, Guski H, Schumacher M. Radiation therapy and tamoxifen after breast-conserving surgery: updated results of a $2 \times 2$ randomised clinical trial in patients with low risk of recurrence. Eur J Cancer. 2010;46(1):95-101.

28. Forrest AP, Stewart HJ, Everington D, Prescott RJ, McArdle CS, Harnett AN, Smith DC, George WD. Randomised controlled trial of conservation therapy for breast cancer: 6-year analysis of the Scottish trial. Scottish Cancer Trials Breast Group. Lancet. 1996;348(9029):708-13.

29. Clarke M, Collins R, Darby S, Davies C, Elphinstone P, Evans V, Godwin J, Gray R, Hicks C, James S, et al. Effects of radiotherapy and of differences in the extent of surgery for early breast cancer on local recurrence and 15-year survival: an overview of the randomised trials. Lancet. 2005;366(9503):2087-106.

30. K S: Breast cancer. Breast cancer: a world of differences. Science (New York, NY) 2014, 343(6178):1452-1453.

31. William J, Gradishar BO, Anderson R, Balassanian SL, Blair HJ, Burstein A, Cyr AD, Elias, William B, Farrar, Andres Forero, Sharon H Giordano et al: Breast Cancer, Version 2.2020, NCCN Clinical Practice Guidelines in Oncology. https://wwwnccnorg 2020.

32. HJ GC, E PW B, MM MGPDSLMC, M P-G R, HJ S et al: De-escalating and escalating treatments for early-stage breast cancer: the St. Gallen International Expert Consensus Conference on the Primary Therapy of Early Breast Cancer 2017. Annals of oncology: official journal of the European Society for Medical Oncology 2019, 30(7):1181.

33. LJ G, C RGSBBHC, S Y. R, S S, FL B, PM R, NE D et al: Prognostic utility of the 21-gene assay in hormone receptorpositive operable breast cancer compared with classical clinicopathologic features. Journal of clinical oncology: official journal of the American Society of Clinical Oncology. 2008;26(25):4063-71.

34. EP M, JP GTBFSPSS, CE CDW, DL G. W, N W: Association between the 21-gene recurrence score assay and risk of locoregional recurrence in node-negative, estrogen receptor-positive breast cancer: results from NSABP B-14 and NSABP B-20. Journal of clinical oncology: official journal of the American Society of Clinical Oncology. 2010;28(10):1677-83. 
35. JA S. S P: Development of the 21-gene assay and its application in clinical practice and clinical trials. Journal of clinical oncology: official journal of the American Society of Clinical Oncology. 2008;26(5):721-8.

36. JA S, RJ G, DF M, KI P, KS A, DF H, CE G, EC D, EA P. JA O et al: Prospective Validation of a 21-Gene Expression Assay in Breast Cancer. N Engl J Med. 2015;373(21):2005-14.

37. Gluz O, Nitz UA, Christgen M, Kates RE, Shak S, Clemens M, Kraemer S, Aktas B, Kuemmel S, Reimer T: West German Study Group Phase III PlanB Trial: First Prospective Outcome Data for the 21-Gene Recurrence Score Assay and Concordance of Prognostic Markers by Central and Local Pathology Assessment. Journal of Clinical Oncology Official Journal of the American Society of Clinical Oncology 2016:JC0.2015.2063.5383.

38. vtV FCLJ, JY JBLSGVSD. P, E B, S C, M D et al: 70-Gene Signature as an Aid to Treatment Decisions in Early-Stage Breast Cancer. N Engl J Med. 2016;375(8):717-29.

39. S O, F P-L ESSK. P P, E R, S Z, F C: Primary breast cancer: ESMO Clinical Practice Guidelines for diagnosis, treatment and follow-up. Annals of oncology: official journal of the European Society for Medical Oncology 2015:v8-30.

40. An X, Lei X, Huang R, Luo R, Li H, Xu F, Yuan Z, Wang S, de Nonneville A, Gonçalves A, et al: Adjuvant chemotherapy for small, lymph node-negative, triple-negative breast cancer: A single-center study and a meta-analysis of the published literature. Cancer 2020:3837-3846.

41. Steenbruggen T, van Werkhoven E, van Ramshorst M, Dezentjé V, Kok M, Linn S, Siesling S, Sonke G: Adjuvant chemotherapy in small node-negative triple-negative breast cancer. European journal of cancer (Oxford, England: 1990) 2020, 135:66-74.

42. NT B-P SI, NA ST, CH T. Y, M S, K K, N B-P: Impact of adjuvant chemotherapy on survival of women with T1NOM0, hormone receptor negative breast cancer. Cancer epidemiology. 2017;48:56-61.

43. HA ASDF, C A S, M P: New strategies in breast cancer: the significance of molecular subtypes in systemic adjuvant treatment for small T1a,bNOM0 tumors. Clinical cancer research: an official journal of the American Association for Cancer Research 2014, 20(24):6242-6246.

44. EO H, AM G-A VV. GN H: Prognosis and management of patients with node-negative invasive breast carcinoma that is $1 \mathrm{~cm}$ or smaller in size (stage $1 ; \mathrm{T} 1 \mathrm{a}, \mathrm{bNOM0}$ ): a review of the literature. Journal of clinical oncology: official journal of the American Society of Clinical Oncology. 2006;24(13):2113-22.

\section{Figures}



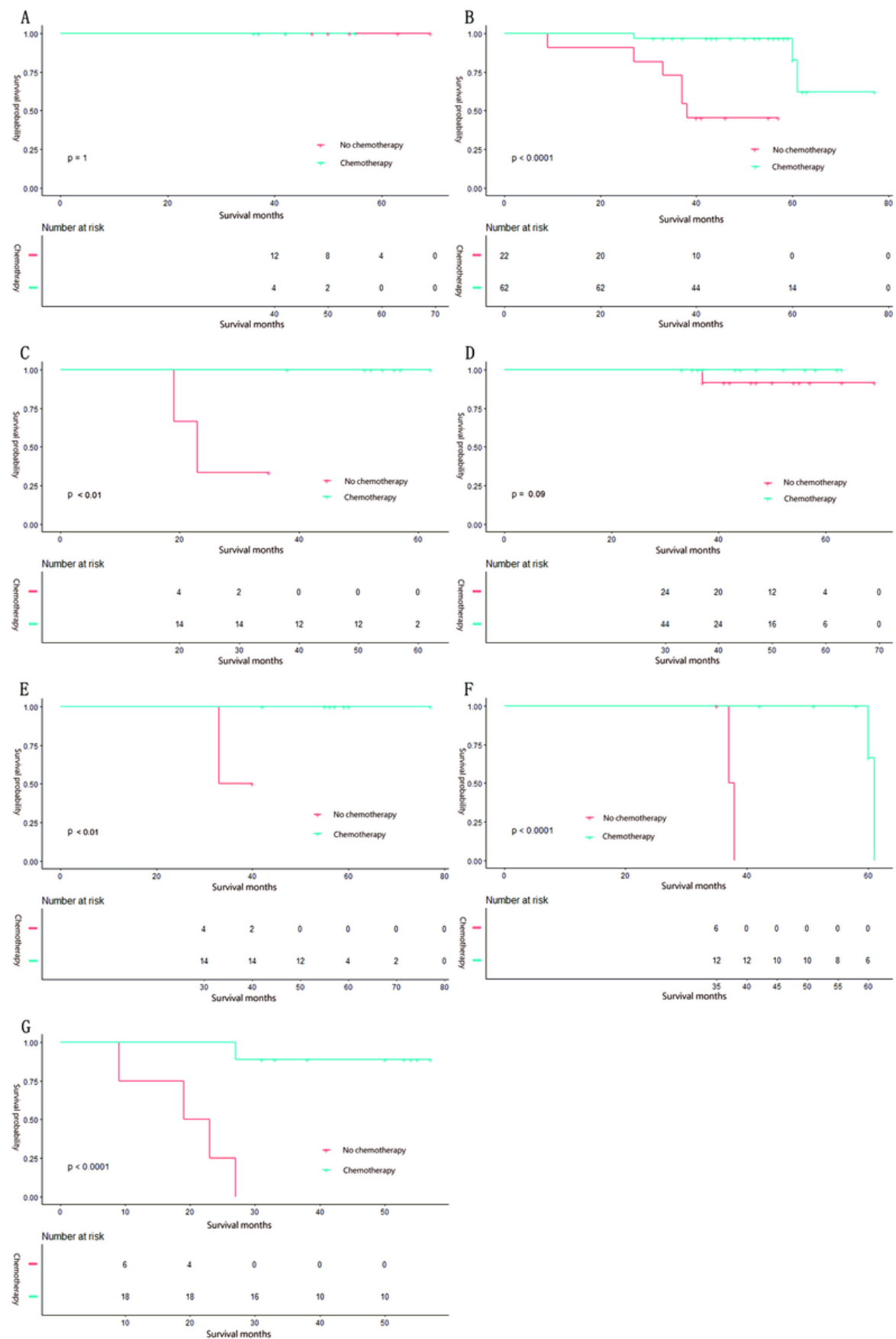

\section{Figure 1}

Kaplan-Meier survival curves of the chemotherapy and no chemotherapy groups according to the grades and molecular subtypes of T1b breast cancer patients treated at Northern Jiangsu People's Hospital. (a) Grade l; (b) grade II; (c) grade III; (d) HR+/HER2-; (e) HR+/HER2+; (f) HR-/HER2+; and (g) HR-/HER2-.

\section{Supplementary Files}

This is a list of supplementary files associated with this preprint. Click to download.

- FigureS1.docx

- Figures2.docx

- S1.tif 
- S2.tif

- supplementaltables.docx

Page 18/18 